\title{
Atração e recrutamento de estudantes internacionais em Portugal: políticas nacionais e institucionais
}

\author{
Cristina Sin, Sónia Cardoso \& Orlanda Tavares
}

\begin{abstract}
Resumo:
O artigo explora as políticas e as estratégias nacionais e institucionais de recrutamento de estudantes internacionais em Portugal, mediante a análise de documentos políticos e de entrevistas semiestruturadas com representantes de duas universidades. Os resultados sugerem uma intensa atividade a nível político, que se concretiza não apenas em mudanças legislativas, mas também na coordenação a nível nacional e na mobilização de um conjunto articulado de organizações que atuam no sentido de aumentar a atratividade de Portugal face aos estudantes estrangeiros. Do ponto de vista institucional, verifica-se, também, que as universidades analisadas são atualmente proactivas no recrutamento destes estudantes, embora ainda não de um modo sistemático e articulado. Aproveitando a vantagem proporcionada pela língua portuguesa, as universidades parecem estar a explorar um nicho de mercado constituído quer pelos estudantes da lusofonia, quer por outros interessados em aprender a língua, sobretudo os estudantes oriundos da China.
\end{abstract}

Palavras-chave:

recrutamento; estudantes internacionais; políticas nacionais; estratégias institucionais. 


\title{
Attracting and recruiting international students in Portugal: national and institutional policies
}

\begin{abstract}
The article explores national and institutional policies and strategies for recruiting international students in Portugal through the analysis of political documents and semi-structured interviews with representatives of two universities. The results point to an intense national political activity, visible not only in legislative changes, but also in the nationwide coordination and mobilisation of an articulated set of organisations to increase Portugal's attractiveness. From an institutional point of view, the analysed universities are now proactive in recruiting international students, although they do not yet do so in a systematic and articulated way. Capitalising on the advantage offered by the Portuguese language, universities seem to be exploring a niche market of Lusophone students and others who are interested in learning Portuguese, especially from China.
\end{abstract}

Keywords: recruitment; international students; national policies; institutional strategies.

\section{Attirer et recruter des étudiants internationaux au Portugal: politiques nationales et institutionnelles}

Résumé: L'article explore les politiques et les stratégies nationales et institutionnelles pour le recrutement d'étudiants internationaux au Portugal à travers l,analyse de documents politiques et des entretiens semi-structurés avec des représentants de deux universités. Les résultats suggèrent une activité politique intense, qui se manifeste non seulement dans des modifications législatives, mais également dans la coordination et dans la mobilisation au niveau national d`un ensemble structuré d`organisations pour accroître l'attractivité du Portugal. Du point de vue institutionnel, on constate que les universités analysées ont commencé à recruter des étudiants étrangers de manière proactive, bien qu'elles ne le fassent pas encore de manière systématique et articulée. Profitant de l'avantage offert par la langue portugaise, les universités semblent explorer un marché de niche d'étudiants lusophones et d'autres intéressés par l'apprentissage du portugais, en particulier de Chine.

Mots-clés: recrutement; étudiants internationaux; politiques nationales; stratégies institutionnelles.

\section{Atraer y reclutar estudiantes internacionales en Portugal: políticas nacionales e institucionales}

Resumen: El artigo explora las políticas y estrategias nacionales e institucionales para reclutar estudiantes internacionales en Portugal a través del análisis de documentos políticos y entrevistas semiestructuradas con representantes de dos universidades. Los resultados apuntan a una intensa actividad política nacional, visible no solo en los cambios legislativos, sino también en la coordinación y movilización a nivel nacional de un conjunto articulado de organizaciones para aumentar la atractividad de Portugal. Desde un punto de vista institucional, las universidades analizadas son ahora proactivas en el reclutamiento de estudiantes internacionales, aunque todavía no lo hacen de manera sistemática y articulada. Aprovechando la ventaja que ofrece el idioma portugués, las universidades parecen estar explorando un nicho de mercado de estudiantes de habla portuguesa y otros interesados en aprender portugués, especialmente de China.

Palabras clave: reclutamiento; estudiantes internacionales; políticas nacionales; estrategias institucionales. 


\section{Introdução}

A geografia dos destinos dos estudantes internacionais encontra-se numa fase de mudança, dado que a competição por estes estudantes se intensificou e passou a envolver um número crescente de países. Conscientes do potencial económico do ensino superior (ES), os países que tradicionalmente mostravam pouco interesse em receber estudantes internacionais em mobilidade de grau começaram a desenvolver políticas para incentivar as suas instituições ao seu recrutamento. A quota de mercado dos quatro maiores recrutadores - Estados Unidos, Reino Unido, Austrália e Canadá - tem diminuído (Choudaha, Chang \& Kono, 2013), ao passo que os destinos de mobilidade se têm diversificado (Börjesson, 2017; França, Alves \& Padilla, 2018).

Países mais pequenos - como Portugal - enfrentam desafios que não se colocam aos países que mais atraem estudantes internacionais (Urbanovič et al., 2016). Primeiro, entraram mais tarde no mercado estudantil internacional, o que significa que o seu ES ainda não tem o reconhecimento mundial dos EUA, do Reino Unido, da Austrália, ou do Canadá (idem). Além disso, recursos financeiros limitados e a falta de uma economia de escala significam, para muitos países, a incapacidade de investir em infraestruturas (por exemplo, alojamento e outras instalações), marketing/branding e recursos humanos. As questões culturais e linguísticas também podem constituir um problema, na medida em que o ensino em inglês coloca os países pequenos num dilema: ser internacional e preservar a sua identidade nacional (idem).

Em Portugal, o recrutamento de estudantes em mobilidade de grau tem assumido destaque na agenda das instituições de ES. Mudanças sociais e económicas, por um lado, e ações políticas, por outro, favoreceram esta nova prioridade: a diminuição do financiamento estatal; o declínio das matrículas e projeções demográficas desfavoráveis (Dias, Mendes, Magalhães \& Infante, 2013); a priorização da mobilidade incoming pela recente estratégia para a internacionalização do ES português (MEC, 2014); e a possibilidade, suscitada por legislação recente, de as instituições públicas cobrarem propinas mais elevadas a estudantes internacionais. Porém, no contexto do ES europeu, e quando comparado a países do Norte da Europa, Portugal surge como menos atraente, devido a uma economia pouco produtiva e competitiva e ao menor investimento em ES e investigação (Fonseca, Esteves \& Iorio, 2015).

$\mathrm{Na}$ recente estratégia de internacionalização, é reconhecida a existência de uma ampla margem de crescimento, enfatizando-se que Portugal é o país mais equilibrado da OCDE em relação aos continentes de origem dos estudantes (exceto Ásia). Atualmente, cerca de dois terços dos estudantes internacionais provêm de países lusófonos. Os estudantes do Brasil têm aumentado consistentemente, enquanto os estudantes de países africanos têm diminuído desde o início do século, devido, em parte, ao desenvolvimento do ES nesses países (Fonseca et al., 2015). Os estudantes europeus 
tendem a provir de países onde as comunidades portuguesas são mais numerosas (Alemanha, França ou Itália) (idem). Porém, o maior crescimento tem-se registado entre os estudantes chineses, o que sugere que a Ásia pode vir a ser uma importante fonte de estudantes internacionais. O recrutamento de estudantes internacionais tem refletido, portanto, a relação privilegiada com os países de língua portuguesa.

Este artigo analisa as políticas e estratégias nacionais e institucionais que visam o recrutamento de estudantes internacionais, num contexto que tem favorecido esta nova prioridade. O objetivo é compreender o modo como Portugal, enquanto país de destino, bem como algumas das suas instituições, atuam de forma a ultrapassar a sua aparente desvantagem no mercado internacional. Dada a natureza exploratória do estudo, não se pretende fazer generalizações para o sistema de ES português. Primeiro, descrevem-se as principais estratégias destinadas a incrementar o recrutamento de estudantes internacionais, conforme reportado pela investigação. De seguida, apresenta-se a metodologia e discutem-se os resultados em dois níveis: o das políticas nacionais e o das políticas institucionais. Por fim, a conclusão elenca as estratégias para atrair estudantes internacionais.

\section{Estratégias para aumentar o recrutamento de estudantes internacionais}

A escolha do país geralmente precede a escolha da instituição na decisão de estudar no estrangeiro (Llewellyn-Smith \& McCabe, 2008). Segundo a OCDE (2016), os estudantes usam quatro critérios para escolher o país: a língua de ensino, a qualidade dos programas, o valor das propinas e a política de imigração. Outros estudos empíricos (Caruso \& de Wit, 2015) identificam outros fatores: os gastos, as perceções sobre segurança, as condições económicas, ou a rede de migrantes no país anfitrião. Perkins e Neumayer (2014) argumentam que os rendimentos nos países de destino, bem como os laços criados por ligações coloniais, a língua comum e a existência prévia de grupos de migrantes têm mais influência na escolha do estudante do que a qualidade do ensino. No caso de países com menor protagonismo no mercado internacional de estudantes, Kondakci (2011) sugere que a proximidade cultural, política e histórica entre os países de origem e de destino determina a dimensão e a direção da mobilidade dos estudantes.

Porém, o recrutamento de estudantes internacionais é da responsabilidade das instituições. Vários estudos consideram-no numa perspetiva de marketing e de concorrência num palco global (Asaad, Melewar, Cohen \& Balmer, 2013; Ross, Grace \& Shao, 2013; Ross, Heaney \& Cooper, 2007). Assim, impõe-se a necessidade de aumentar a visibilidade da instituição através da criação de uma marca - branding. Choudaha et al. (2013) realçam a importância de uma estratégia holística de recrutamento que englobe um amplo leque de práticas que passam pelos modelos tradicionais de 
participação em feiras, ou visitas internacionais de recrutamento, até à contratação de agentes. Os autores identificam três categorias de práticas emergentes: tecnologias para alcançar mais estudantes de um modo rentável; parcerias para criar visibilidade e canais de recrutamento; e investigação para ajudar na priorização de esforços e na medição do retorno do investimento. Em relação às tecnologias, o website continua a ser uma poderosa ferramenta, complementada com feiras universitárias virtuais, apresentações web, webinars, vídeos, chats, chamadas Skype, blogs, etc.

Na mesma linha, Asaad et al. (2013) sublinham a importância de as instituições terem consciência da complexidade do contexto internacional, o que implica fazer estudos de mercado através de inquéritos, entrevistas ou a participação em feiras internacionais para obter informação e avaliar as diferentes necessidades dos potenciais candidatos Ross et al. (2007) realçam a importância de as instituições terem não só uma estratégia de marketing internacional, como também um departamento de marketing, dotado de pessoal que domine o conceito e seja capaz de aplicá-lo no âmbito do recrutamento internacional.

A facilidade de interação dos estudantes internacionais com a instituição, logo de início (mas também mais tarde), é crucial para a sua capacidade de atração. De acordo com Russell (2005), o tipo de serviço a que os estudantes têm acesso - o nível de burocracia e de complexidade - pode influenciar a decisão de candidatura, bem como a satisfação após a inscrição. A satisfação gera publicidade positiva, com os próprios estudantes a promoverem a instituição. A disponibilização de informações consistentes e precisas concorrem para minimizar a perceção dos obstáculos à entrada e, por conseguinte, para aumentar as matrículas, o que, por sua vez, concede à instituição uma vantagem competitiva relativamente às demais (Ross et al., 2013).

Constituem exemplos de estratégias de atração de estudantes internacionais usadas por instituições de países de menor protagonismo: a participação em feiras e encontros estudantis, a criação de estruturas administrativas de apoio aos estudantes através da contratação de pessoal dedicado a trabalhar com este público (Kubiciel-Lodzinska \& Ruszczak, 2016) ou a criação de serviços para estudantes internacionais (Cox, 2012). Algumas instituições simplificaram os procedimentos de recrutamento e ofereceram apoio aos estudantes internacionais para legalizar a sua permanência no país de acolhimento (Kubiciel-Lodzinska \& Ruszczak, 2016). As instituições recorrem ainda à tecnologia (web e media digitais) e a novas parcerias (Cox, 2012) entre uma instituição de ES e escolas secundárias, instituições de ES estrangeiras, agências governamentais ou outras organizações, no sentido de melhorar a visibilidade e assegurar um fluxo constante de estudantes.

Em Portugal, antes do lançamento da estratégia nacional de internacionalização (2014), as instituições já recrutavam estudantes internacionais, embora através de iniciativas frequentemente marginais e ad hoc (Rosa et al., 2004; Veiga et al., 2006). 
As instituições públicas não sentiam necessidade de recrutar estudantes internacionais além dos que provinham dos países lusófonos patrocinados por subsídios do governo português, e que eram incluídos na fórmula de financiamento em pé de igualdade com os estudantes nacionais. Pelo contrário, as instituições privadas estavam ativas no recrutamento de estudantes internacionais, dadas as mais-valias financeiras que esse recrutamento representava num contexto de perda progressiva e acentuada de candidatos nacionais. Um estudo sobre as estratégias de internacionalização de dez instituições públicas (Guerreiro, 2015) identifica algumas iniciativas que visam atrair estudantes internacionais: a oferta de um 'ano zero' para facilitar a integração e a aprendizagem do português; cursos oferecidos no idioma nativo dos estudantes (francês, italiano ou inglês) ou campanhas de marketing internacional. As duas primeiras revelam uma intenção de ampliar a rede de recrutamento para além dos estudantes dos países lusófonos. O estudo identifica, ainda, a Ásia como um "mercado apetecível", embora assinale algumas limitações neste âmbito, nomeadamente a lentidão dos regulamentos sobre vistos e residências ou a falta de articulação entre o Estatuto do Estudante Internacional e o Serviço de Estrangeiros e Fronteiras. De acordo com os líderes institucionais, as restrições financeiras impostas às instituições constituem um obstáculo para a implementação de uma estratégia de marketing internacional, a contratação de pessoal qualificado em número suficiente e a prestação de apoio necessário aos estudantes internacionais. A língua emerge como outro desafio: se, por um lado, as instituições dão sinais de querer explorar o potencial do português como uma das línguas mais faladas no mundo, por outro, parecem querer promover o inglês como segunda língua, ainda que com algumas reservas, quer por parte do corpo docente, quer dos estudantes (Guerreiro, 2015).

\section{Metodologia}

Este estudo baseou-se na análise de legislação, resoluções ou outros documentos emitidos por órgãos governamentais e outras organizações relevantes. Os documentos incluem:

i. Estratégia para a internacionalização do ensino superior português (2014).

ii. Decreto-Lei 36/2014: Estatuto do Estudante Internacional (2014).

iii. Resolução do Conselho de Ministros n. ${ }^{\circ}$ 47/2015.

iv. Resolução do Conselho de Ministros n. ${ }^{\circ}$ 78/2016.

v. PortugalGlobal: A crescente internacionalização do ensino superior português. (2017). AICEP (Agência Para o Investimento e Comércio Externo). 
Os documentos selecionados foram submetidos a uma análise documental que se centrou em três temas fundamentais: as circunstâncias que justificaram a crescente importância do recrutamento dos estudantes internacionais, os instrumentos utilizados para aumentar a atratividade e os principais atores envolvidos. Desta análise resultou a identificação das políticas nacionais de recrutamento, apresentadas na primeira secção dos resultados.

O estudo baseou-se, também, na análise de conteúdo de entrevistas semiestruturadas com representantes de duas universidades portuguesas (ver Quadro 1). As entrevistas foram realizadas em 2017, com representantes institucionais em cargos de gestão e tomada de decisão relacionados com o recrutamento de estudantes internacionais. Os entrevistados pertenciam a duas universidades públicas do norte de Portugal (quatro entrevistas em cada) (Quadro 1).

Quadro 1. Representantes institucionais entrevistados

\begin{tabular}{|l|l|}
\hline Universidade A & Vice-Reitor para a internacionalização \\
& Vice-Reitor para os assuntos académicos \\
& Diretor do gabinete de relações internacionais \\
Diretor dos serviços académicos \\
\hline Assessor do Reitor em assuntos de internacionalização \\
Universidade B & Diretor do gabinete de relações internacionais \\
& Diretor dos serviços académicos \\
& Diretor do gabinete de comunicação e imagem \\
\hline
\end{tabular}

Fonte: autoras

A seleção de instituições públicas prendeu-se com o facto de o recrutamento de estudantes internacionais se ter tornado, apenas recentemente, uma prioridade para estas instituições. Por razões logísticas, a escolha recaiu sobre duas universidades no norte de Portugal, com dimensões e idades diferentes, para verificar se tais características institucionais dão origem a abordagens e dificuldades diferentes. O termo "estudantes internacionais" aplica-se, no contexto do estudo, a todos os estudantes de países estrangeiros, exceto os países da União Europeia e do Espaço Económico Europeu (a estes últimos estudantes não podem ser cobradas propinas diferentes da dos estudantes nacionais por imposições legais europeias).

As entrevistas foram gravadas, transcritas e analisadas de acordo com o método da teoria fundamentada nos dados (grounded theory), adaptada de Strauss e Corbin (1990). Este método envolveu uma codificação aberta e uma codificação seletiva. No primeiro caso, as entrevistas foram divididas em segmentos codificados para gerar categorias. Os códigos e as relações entre as categorias foram constantemente 
cruzados com os dados para garantir a sua consistência. A codificação seletiva permitiu gerar as categorias definitivas e o quadro analítico final, o qual se organizou em torno das categorias: branding institucional, revisão de procedimentos de admissão e adaptação curricular.

\section{Resultados: atração e recrutamento de estudantes internacionais em Portugal}

Nesta secção, apresentam-se os resultados decorrentes da análise documental (Políticas nacionais) e da análise de conteúdo das entrevistas (Políticas institucionais: as estratégias de duas universidades portuguesas).

\section{Políticas nacionais}

Quanto às circunstâncias que justificaram a crescente importância atribuída ao recrutamento de estudantes internacionais, é possível destacar desde logo o Processo de Bolonha que suscitou uma maior consciência da necessidade de internacionalização e do potencial de atração do espaço europeu. Porém, o que mais parece ter realçado esta importância foi a tentativa política de alinhamento do ES português com as tendências de globalização da ciência e da tecnologia, refletidas em todos os documentos analisados. A estratégia nacional para a internacionalização do ES representou o ponto de viragem em termos da prioridade atribuída à política de recrutamento (MEC, 2014). Liderada pelos Ministérios do Desenvolvimento Regional e da Educação e Ciência, esta estratégia veio preencher uma lacuna política na orientação nacional e na coordenação das atividades das instituições neste domínio. A internacionalização do ES português era "difusa, mal articulada e com resultados modestos, quando comparada com os recursos investidos, já que muitas instituições realizam as suas atividades de forma isolada" (idem, 11-12). O recrutamento de estudantes internacionais surge como uma das dimensões principais da estratégia, cujo objetivo é o de duplicar o número destes até 2020.

Os instrumentos para aumentar a capacidade de atração de estudantes internacionais podem ser de três tipos: informação/persuasão, regulação e mecanismos económicos (Vedung, 1998). A estratégia nacional pertence ao primeiro tipo, dado que tece uma série de recomendações a vários níveis: promover o país e as suas instituições; criar estratégias de cooperação com regiões do mundo específicas (além dos países da CPLP); aumentar a oferta de educação em inglês; promover uma melhor e mais abrangente prestação de informações aos potenciais candidatos; simplificar os processos burocráticos para obter vistos, alojamento, números fiscais, etc., por intermédio da cooperação com entidades públicas (por exemplo, o Serviço de Estrangeiros e Fronteiras); ou criar uma 'via verde' para a admissão de estudantes internacionais. 
As mudanças legislativas, também em 2014, representam um instrumento de regulação. Nos anos anteriores, a legislação já tinha contribuído para a melhoria das condições dos estudantes estrangeiros, pela criação de um "cartão azul UE" para facilitar a permanência de estrangeiros altamente qualificados, bem como pela simplificação dos procedimentos de residência (Fonseca et al., 2015). No entanto, o diploma que marcou a diferença foi o Estatuto do Estudante Internacional, de 2014, que definiu um novo regime de entrada para estes estudantes independente dos requisitos de admissão aplicáveis aos estudantes portugueses. O diploma criou também a possibilidade de as instituições públicas cobrarem propinas mais altas aos estudantes internacionais (fora da UE), considerando o custo real da educação. No entanto, foi mantido o regime especial anterior para a admissão de estudantes que beneficiam de bolsas de estudo do governo português e que são provenientes dos países africanos de língua portuguesa e de Timor-Leste, mantendo-se a relação privilegiada com estes países. Espera-se que este estatuto resulte em estratégias institucionais de recrutamento mais coordenadas, incluindo uma diversificação dos países-alvo. Em 2016, a Resolução 78/2016 mandatou a Direção-Geral do Ensino Superior, em estreita articulação com as instituições de ES e a Agência Portuguesa para o Investimento e Comércio Externo (AICEP), de divulgar a oferta educativa das instituições portuguesas através do portal Study \& Research in Portugal (www.study-research.pt).

Por último, o Estatuto do Estudante Internacional representa também um mecanismo económico, enquanto instrumento político. Abrindo a possibilidade às instituições de ES de cobrarem propinas mais elevadas, o estatuto conduziu a estratégias institucionais de recrutamento mais concertadas, que contemplam a diversificação dos países alvo de recrutamento para além dos países tradicionais de língua portuguesa. Tal como sublinhado pelo presidente da comissão de internacionalização do Conselho de Reitores das Universidades Portuguesas (CRUP), "há uma mentalidade para mudar e uma ideia a ter em conta: o ensino superior é exportável” (Assunção, 2017, p.7). Nesta lógica, coordenadas pelo CRUP, as quinze universidades públicas criaram a iniciativa "Universidades Portugal" para se tornarem intervenientes globais. Na mesma linha, convocaram o apoio de outros atores (Governo, Instituto Camões, AICEP, Turismo de Portugal, embaixadas, etc.), definiram mercados-alvo prioritários (Angola, Brasil, China, Colômbia, Equador, Luxemburgo, Macau, Moçambique e Peru), obtiveram financiamento da UE e definiram um cronograma de marketing e promoção nos países-alvo (Assunção, 2017).

No que diz respeito aos atores, houve uma tomada de consciência de que as instituições de ES não seriam as únicas responsáveis pelo recrutamento de estudantes internacionais. Esta capacidade é também condicionada por questões relacionadas com políticas de migração ou com assuntos consulares e políticos, não necessariamente alinhados com as intenções das instituições. Portanto, a responsabilidade pelo 
sucesso do recrutamento é partilhada por vários atores. Neste contexto, a estratégia de internacionalização teve também por objetivo lidar com a "grande desarticulação entre os diversos setores da Administração Pública e das instituições envolvidas" (MEC, 2014, p. 10). Quer a estratégia, quer a Resolução 78/2016, quer ainda a própria atuação do CRUP, contemplam uma variedade de atores com diversos papéis e responsabilidades em termos de promoção do recrutamento de estudantes internacionais (e.g. Ministério dos Negócios Estrangeiros, Serviço de Estrangeiros e Fronteiras, AlCEP, Fundação para a Ciência e a Tecnologia, Direção-Geral do Ensino Superior, Alto Comissariado para as Migrações, etc.).

\section{Políticas institucionais: as estratégias de duas universidades portuguesas}

Embora a expetativa fosse a de que os atores pudessem ter perceções diferentes sobre as políticas institucionais, consoante a idade e a dimensão das instituições, tal não se verificou, exceto no caso particular assinalado abaixo (estrutura organizacional da Universidade A). No que diz respeito às duas universidades em análise, os dados recolhidos sugerem que nenhuma parecia ter políticas ou estratégias integradas e articuladas de recrutamento de estudantes internacionais. Os entrevistados reconheceram amiúde a necessidade de mais planeamento e coordenação, por oposição a medidas ad hoc para aumentar o recrutamento. Além disto, a definição de uma estratégia institucional é ainda dificultada, na Universidade A, pela estrutura organizacional em faculdades com um elevado grau de autonomia e com diferentes expectativas, capacidades e experiências em termos do acolhimento de estudantes internacionais.

O recrutamento de estudantes internacionais foi salientado pelos entrevistados como uma prioridade recente, impulsionada pelo Estatuto do Estudante Internacional e os incentivos financeiros nele implícitos, bem como pela ameaça representada pela redução demográfica (Dias et al., 2013). O fluxo de estudantes internacionais foi, muitas vezes, referido como uma consequência de colaborações internacionais pré-existentes de académicos e/ou faculdades, o que é indicador da novidade que o recrutamento constituía enquanto área estratégica. Portanto, na opinião dos entrevistados, o maior desafio era representado pela criação de uma estrutura institucional com um mandato e competência especificamente dedicados ao recrutamento destes estudantes e ao apoio dos mesmos na sua interação com a instituição, desde a candidatura até à conclusão dos estudos. Esta estrutura deveria também centralizar e garantir a comunicação clara e consistente de toda a informação necessária a estes estudantes em diferentes momentos.

Apesar da falta de uma estratégia institucional formal, ambas as universidades pareciam ter implementado um número considerável de iniciativas que, embora coordenadas centralmente, seriam iniciativas sobretudo ad hoc. Coincidentes com algumas das iniciativas identificadas na literatura (Russell, 2005; Kubiciel-Lodzińska \& Ruszczak, 
2016; Choudaha et al., 2013), as adotadas pelas duas universidades dividiam-se em três categorias - branding institucional; revisão dos procedimentos de admissão; e adaptação curricular - sendo as duas primeiras mais predominantes do que a terceira.

As iniciativas de branding institucional promovidas pelas duas instituições consistiam na intensificação da participação em feiras internacionais, no estabelecimento de convénios e parcerias com escolas e instituições de países-alvo, na produção de material promocional, no reforço da sua presença nas redes sociais e nas visitas a escolas secundárias, ou outras organizações, nos países-alvo. Como esperado, os principais países-alvo eram os países lusófonos (com a prevalência do Brasil, seguido pelos países africanos - Cabo Verde, Angola e Moçambique). No entanto, a Ásia surgiu como um mercado muito atraente para ambas as instituições, especialmente a China e, em menor grau, a Índia e a Indonésia. Na América Latina, além do Brasil, foram também mencionados a Colômbia e o Peru. Outra ambição visava o recrutamento não apenas de mais, mas também de melhores estudantes numa tentativa de melhorar a reputação da instituição no exterior.

Com o propósito de rever os procedimentos de admissão, as instituições procederam à clarificação e simplificação das informações necessárias à candidatura destes estudantes, por exemplo quem poderia usufruir do estatuto de estudante internacional. Para atender aos interesses dos estudantes de países onde os anos académicos têm uma estrutura diferente, as instituições reviram o cronograma de candidatura (adaptado, até então, ao cronograma dos estudantes nacionais) para permitir que a mesma se pudesse efetuar em diferentes alturas do ano, concedendo tempo suficiente para os estudantes tratarem das formalidades necessárias à sua mudança para Portugal. Uma última medida promovida para facilitar a admissão consistia no reconhecimento automático dos exames de conclusão do ensino secundário nos principais países-alvo (Brasil e China). Estas medidas estão em consonância com as recomendações para minimizar a complexidade e a burocracia (Russell, 2005; Ross et al., 2013).

Os entrevistados de ambas as instituições referiram, ainda, a realização de descontos nas propinas para os candidatos dos países lusófonos para fomentar a sua atratividade. A adoção desta estratégia pode ser, eventualmente, explicada por uma tentativa de incentivar a preservação das relações históricas e culturais com esses países (França et al., 2018).

Relativamente à adaptação curricular, verificaram-se sobretudo preocupações com a língua usada no ensino/aprendizagem. Uma prática recorrente consistia em facultar aos estudantes internacionais aulas de português. A Universidade B criou um 'ano zero' para facilitar a aprendizagem da língua antes da frequência do ciclo de estudos (Guerreiro, 2015). O ensino em inglês surgiu como pouco frequente ao nível do primeiro ciclo (onde se aplica o Estatuto do Estudante Internacional), embora o fosse ao nível do segundo e terceiro ciclos. Isso pode dever-se a uma certa relutância, quer de 
docentes, quer de estudantes, em usar outra língua de ensino que não o português (Guerreiro, 2015; Pinto e Araujo e Sá, 2018) ou a uma perceção institucional, segundo a qual a língua portuguesa tem vantagens no mercado internacional.

Esta vantagem é reconhecida não apenas no caso dos estudantes de países lusófonos, mas também de outros países, interessados em aprender a língua dado o seu potencial como uma das línguas mais faladas no mundo. Na China valoriza-se o português como facilitador das relações comerciais com países de língua oficial portuguesa. Embora reconhecido como pouco frequente, o ensino em inglês constituía um desafio segundo os entrevistados, na medida em que poderia contribuir para ampliar a área de recrutamento internacional das instituições para além dos países lusófonos. Porém, e no sentido de evitar um eventual impacto negativo do ensino em inglês no recrutamento de estudantes de países de língua portuguesa, os entrevistados propunham que os cursos pudessem ser facultados, quer em português, quer em inglês. Contudo, a capacidade do corpo docente para ensinar em inglês foi reconhecida como um outro desafio.

\section{Conclusão}

Este artigo teve por objetivo analisar as políticas e estratégias nacionais e de duas instituições de ES destinadas ao recrutamento de estudantes internacionais e discutir o modo como estas pretendem colmatar as desvantagens de Portugal em relação, quer aos principais recrutadores mundiais, quer aos países mais ricos da Europa.

Os últimos anos foram marcados por uma intensa atividade dos decisores políticos nacionais que têm vindo a implementar uma série de instrumentos: legislação facilitadora da matrícula de estudantes internacionais, incentivos financeiros, um quadro nacional de ação e iniciativas de marketing. Vários atores têm sido chamados a contribuir para a promoção do país e do seu ES. A colaboração da AICEP, bem como a possibilidade de estabelecer propinas mais elevadas como incentivo às instituições na atração de um maior número de estudantes internacionais, traduzem uma perceção crescente do ES como uma mercadoria transacionável. As medidas ao nível nacional parecem ter dado ímpeto às instituições que dão indícios de estarem a mobilizar-se para recrutar estudantes internacionais, adotando um conjunto de medidas e pensando o recrutamento para além dos públicos tradicionais.

É possível afirmar que as duas instituições analisadas se têm tornado mais proactivas no recrutamento de estudantes internacionais, ainda que, em grande parte, dependentes da vantagem representada pela língua portuguesa. Os países lusófonos continuam a representar o principal público-alvo, devido aos laços históricos reforçados pelo estabelecimento da comunidade dos países de língua portuguesa (França et al., 2018). Dada a mais-valia representada pela língua portuguesa, as instituições 
parecem estar a explorar um nicho de mercado, ampliando a área de recrutamento além dos seus públicos tradicionais (principalmente à China), e a posicionar-se como um concorrente emergente. As estratégias institucionais adotadas no âmbito da revisão dos procedimentos de admissão também parecem reforçar a preferência por dois públicos específicos: os estudantes de países de língua portuguesa e os estudantes chineses. Porém, o grande desafio é constituído pela implementação de estruturas institucionais capazes de providenciar um apoio contínuo e consistente aos estudantes internacionais nas diferentes fases de interação com a instituição (Ross et al., 2013; Russel, 2005). Um outro desafio seria a consolidação de estudos de mercado como instrumento de auxílio à tomada de uma decisão informada sobre as regiões-alvo.

Embora o estudo se limite à análise de duas universidades públicas, os resultados podem ser indicativos das tendências das instituições públicas portuguesas em termos do recrutamento de estudantes internacionais, dado parecerem replicar a evolução identificada por outras análises (Veiga et al., 2006). Investigações futuras poderiam alargar o presente estudo a mais instituições, nomeadamente as politécnicas e as privadas.

\section{Referências Bibliográficas}

Asaad, Y., Melewar, T. C., Cohen, G., \& Balmer, J. (2013). Universities and export market orientation: An exploratory study of UK post-92 universities. Marketing Intelligence \& Planning, 31(7), 838-856.

Assunção, M. (2017). Exportação do ensino superior. In PortugalGlobal: A crescente internacionalização do ensino superior português (pp. 7-8). Lisboa: AICEP.

Börjesson, M. (2017). The global space of international students in 2010. Journal of Ethnic and Migration Studies, 43(8), 1256-75.

Caruso, R., \& de Wit, H. (2015). Determinants of mobility of students in Europe: Empirical evidence for the period 1998-2009. Journal of Studies in International Education, 19(3), 265-282.

Choudaha, R., Chang, L., \& Kono, Y. (2013). International student mobility trends 2013: Towards responsive recruitment strategies. New York: World Education Services.

Cox, M. (2012). International student recruitment: policies and developments in selected countries: Sweden, Norway and Finland. The Hague: Nuffic.

Dias, R., Mendes, M.F., Magalhães, M.G., \& Infante, P. (2013, October). The role of population projections for a redefinition of the Portuguese higher educational institutional network. Paper apresentado na Joint Eurostat -UNECE-ISTAT Work Session in Demographic Projections, Rome.

Fonseca, M. L., Esteves, A., \& lorio, J. (2015). Mobilidade internacional de estudantes do ensino superior: os alunos universitários brasileiros em Portugal. In J. Peixoto, B. Padilla, J.C. Marques \& P. Góis (Orgs.), Vagas atlânticas: migrações entre Brasil e Portugal no início do século XXI (pp. 149175). Lisboa: Editora Mundos Sociais. 
França, T., Alves, E., \& Padilla, B. (2018). Portuguese policies fostering international student mobility: a colonial legacy or a new strategy? Globalisation, Societies and Education, 1-14.

Guerreiro, C. (2015). A internacionalização do ensino superior português as razões, as estratégias e os desafios (Dissertação de mestrado). Porto: Instituto Politécnico do Porto.

Kondakci, Y. (2011). Student mobility reviewed: Attraction and satisfaction of international students in Turkey. Higher Education, 62(5), 573.

Kubiciel-Lodzińska, S., \& Ruszczak, B. (2016). The determinants of student migration to Poland based on the Opolskie Voivodeship Study. International Migration, 54(5), 162-174.

Llewellyn-Smith, C., \& V.S. McCabe. (2008). What is the attraction for exchange students: the host destination or the host university? Empirical evidence from a study of an Australian University. International Journal of Tourism Research, 10, 593-607.

Ministério da Educação e Ciência. (2014). Uma estratégia para a internacionalização do Ensino Superior Português. Lisboa: MEC.

OECD (2016). Education at a Glance. OECD Indicators. Paris: OECD.

Perkins, R., \& Neumayer, E. (2014). Geographies of educational mobilities: Exploring the uneven flows of international students. The Geographical Journal, 180(3), 246-259.

Pinto, S., \& Araújo e Sá, M. H. (2019). Language education policy in Portuguese public universities: The voices of institutional stakeholders. Current Issues in Language Planning, 20(2), 140-159.

Ross, M., Grace, D., \& Shao, W. (2013). Come on higher ed... get with the programme! A study of market orientation in international student recruitment. Educational Review, 65(2), 219-240.

Ross, M., Heaney, J.G., \& Cooper, M. (2007). Institutional and managerial factors affecting international student recruitment management. International Journal of Educational Management, 21(7), 593605.

Russell, M. (2005). Marketing education: A review of service quality perceptions among international students. International Journal of Contemporary Hospitality Management, 17(1), 65-77.

Strauss, A., \& J. M. Corbin. (1990). Basics of qualitative research: Grounded theory, procedures and techniques. Thousand Oaks, CA: Sage.

Urbanovič, J., Wilkins, S., \& Huisman, J. (2016). Issues and challenges for small countries in attracting and hosting international students: the case of Lithuania. Studies in Higher Education, 41(3), $491-$ 507.

Vedung, E. (1998). Policy instruments: Typologies and theories. In M.-L. Bemelmans-Videc, R.C. Rist \& E. Vedung (Orgs.), Carrots, sticks and sermons: Policy instruments and their evaluation (pp. 21-58). New Brunswick and London: Transaction Publishers.

Veiga, A., Rosa, M., \& Amaral, A. (2006). The internationalisation of Portuguese higher education: how are higher education institutions facing this challenge? Higher Education Management, 18(1), 113-28. 
Cristina Sin

Agência de Avaliação e Acreditação do Ensino Superior (A3ES) Centro de Investigação de Políticas de Ensino Superior (CIPES), Portugal

Email: cristina.sin@a3es.pt Orcid: https://orcid.org/0000-0001-6039-8194

Sónia Cardoso

Agência de Avaliação e Acreditação do Ensino Superior (A3ES) Centro de Investigação de Políticas de Ensino Superior (CIPES), Portugal

Email: sonia.cardoso@a3es.pt Orcid: https://orcid.org/0000-0001-6504-8468

Orlanda Tavares

Agência de Avaliação e Acreditação do Ensino Superior (A3ES) Centro de Investigação de Políticas de Ensino Superior (CIPES), Portugal

Email: orlanda.tavares@a3es.pt Orcid: https://orcid.org/0000-0002-8750-2032

Correspondência Cristina Sin Agência de Avaliação e Acreditação do Ensino Superior Praça de Alvalade, 6 - 5. ${ }^{\circ}$ Frente 1700-036 Lisboa, Portugal

Data de submissão: Março 2019

Data de avaliação: Maio 2019

Data de publicação: Abril 2020 